\section{Life satisfaction and frailty among older adults}

\author{
Katarina Wilhelmson,, 1,2 Emelie Fritzell,1 \\ Kajsa Eklund,2,3
}

Synneve Dahlin-Ivanoff2,3

1Department of Public Health and Community Medicine/Social Medicine, Sahlgrenska Academy at University of Gothenburg, Gothenburg;

2Vårdalinstitutet, Swedish Institute

for Health Sciences, University of

Gothenburg and Lund; 3Department of Clinical Neuroscience and Rehabilitation, Sahlgrenska Academy at University of Gothenburg, Gothenburg, Sweden

\section{Abstract}

Functional and physical impairment are factors believed to lead to declined life satisfaction among older adults. This study aimed to examine life satisfaction among older adults and the influence of frailty. Baseline data from two studies addressing frail older adults aged $80+$ in Gothenburg, Sweden, $(n=577)$ were used. Frailty was measured through eight indicators. Life satisfaction was measured with Fugl-Meyer's instrument LiSat-11. Perceived life satisfaction was rather high within the studied population, with $66 \%$ being satisfied with life as a whole. Most life satisfaction items were significantly associated with frailty status, with non-frail participants being satisfied to a higher extent for all items with the exception of financial situation, sexual life and partnership relation. The factors significantly explaining life satisfaction were psychological health, partner relationship, leisure and ADL. This study shows that older adults' satisfaction with life as a whole is almost as high as in younger age groups. Respondents with higher degree of frailty reported significantly lower degrees of life satisfaction, indicating a possibility to maintain life satisfaction by preventing or delaying the development of frailty.

\section{Introduction}

Frailty is a geriatric syndrome caused by the multi-system deterioration in reserve capacity at advanced ages. ${ }^{1}$ A review article on frailty showed that the concept most commonly included the following factors; mobility, balance, muscle strength, motor processing, cognition, nutrition, endurance and physical activity. ${ }^{2}$ Frailty can be regarded as a dynamic concept ranging from not being frail to being frail, and it is regarded as distinct from but strongly linked to restricted activity and morbidity. ${ }^{1}$ Results from earlier research suggest that frailty can have negative impact on a variety of quality of life domains. ${ }^{3}$ With the use of labels as frailty, there is a risk of adding to the negative stereotypes and attitudes of aging. 4 Since these stereotypes might affect the quality of life of older adults, there is a need to examine this empirically.

Functional and physical impairment are factors believed to lead to declined life satisfaction. ${ }^{5}$ Even though the concept of life satisfaction has been of interest in geriatric research for a long time, research about life satisfaction among older adults whom experience frailty has been sparse. 6

Life satisfaction derives from an overriding concept of being on the positive end of the continuum of psychological wellbeing. The concept of life satisfaction is based on a subjective experience of life as a whole, ${ }^{7}$ and can be based on affective and rational aspects of life, which in turn are weighted by the individual. ${ }^{8}$ The rational aspect of life satisfaction has also been described in rehabilitation research as the ability to reach individual goals. ${ }^{9}$

Previous research has shown that life satisfaction was higher among older adults of lower age, being male and who were living in ordinary housing. Higher life satisfaction was also reported among older adults that had a higher self-care capacity, a better perceived overall health, higher level of physical activity and that had better economic resources. ${ }^{6}$ In a Danish study frail older adults were more likely to express life satisfaction when they were occupied as usual, had friends, felt able to manage their own lives, did not live alone and if they not recently had lost a close friend. 10 There is reason to believe that frailty may affect selfperceived life satisfaction since frailty affects physical health and the ability to maintain physical and social living pattern.

\section{Objective}

This study aimed to analyze life satisfaction within an older population (80+), and to investigate to what extent frailty affects the experience of life satisfaction.

\section{Materials and Methods}

The study was a cross sectional study and was part of the research program Support for frail elderly persons- from prevention to palliation in Gothenburg, Sweden (http:/www. vardalinstitutet.net). Baseline data from two of the intervention studies within the program,11,12 were used for analyses. The studies have been approved by the regional Ethical Review Board in Gothenburg (ref. no: 650-07, ref. no: 413-08).
Correspondence: Katarina Wilhelmson,

Sahlgrenska Academy at University of Gothenburg, Department of Public Health and Community Medicine/Social Medicine, P.O Box 453, SE 40530 Göteborg, Sweden.

Tel. +46.317 .866 .873 - Fax: +46.311 .62847$

E-mail: katarina.wilhelmson@socmed.gu.se

Key words: life satisfaction, aged 80 and over, frailty, Fugl-Meyer's LiSat-11.

Acknowledgements: this work was supported by Vårdalinstitutet, The Swedish Institute for Health Sciences (ClinicalTrials.gov registration number: NCT0087705 and NCT01260493).

Contributions: KW, KE, SDI have made substantial contributions to the conception and design; $\mathrm{KW}$, EF has been responsible for analysis and interpretation of the data and drafting the article; $\mathrm{KE}$, SDI has been responsible for critical revision of the article and made substantial contributions to the interpretation of the data.

Conflict of interests: the authors report no potential conflict of interests.

Received for publication: 21 March 2013.

Revision received: 27 May 2013.

Accepted for publication: 2 June 2013.

This work is licensed under a Creative Commons Attribution NonCommercial 3.0 License (CC BYNC 3.0).

CC Copyright K. Wilhelmson et al., 2013

Licensee PAGEPress, Italy

Health Psychology Research 2013; 1:e32 doi:10.4082/hpr.2013.e32

\section{Sample}

The sample consists of people 80 years or older, living in ordinary housing. One part of the sample was independent of help from another person in activities of daily living and cognitively intact (assessed by Mini Mental State Examination), ${ }^{13}$ retrieved from two municipalities in Gothenburg, using the official register of all people over 80 years old, $\mathrm{n}=459.11$ The other part was retrieved from the Accident and Emergency department of Mölndal Hospital, and consisted of people discharged back to their ordinary housing, with exclusion of people needing immediate assessment or treatment by a physician, obvious cognitive impairment or palliative care, $\mathrm{n}=127.12$ Using these two parts resulted in a wider spectrum of frailty within the sample. All data collection was performed in the respondent's home using a study questionnaire. Nine persons were excluded from this study because of missing data concerning life satisfaction, which remained a sample of 577 persons. 


\section{Life satisfaction}

Fugl-Meyers Life Satisfaction Assessment (LiSat-11) was used to measure life satisfaction. LiSat-11 consists of eleven items; the first was an overriding question on satisfaction with life as a whole. The following ten items represented different domains in life, which were vocational situation, financial situation, leisure, contacts with friends, sexual life, activities of daily living (ADL), family life, partnership relation, physical health and psychological health. ${ }^{9}$ The respondents were asked to estimate to what extent they experienced satisfaction within each item on a six-grade scale, ranging from very dissatisfied to very satisfied. The LiSat-11 has been validated for Swedish adults (men and women aged 18-74 years), and has adequate test-retest, discriminate and specificity validities. ${ }^{9}$ In the analysis, the sixgrade scale were categorized into satisfied (very satisfied and satisfied), rather satisfied and dissatisfied (very dissatisfied, dissatisfied and rather dissatisfied), as it was done in the validation of the LiSat-11.9

\section{Frailty}

The degree of frailty was measured by seven core frailty indicators; weakness, fatigue, weight loss, low physical activity, poor balance, gait speed and visual impairment.11,12 Cut off points for frailty used in the study were for weakness; grip strength below $13 \mathrm{~kg}$ for women and below $21 \mathrm{~kg}$ for men on the right hand, and below $10 \mathrm{~kg}$ respectively $18 \mathrm{~kg}$ on the left hand. ${ }^{14}$ Fatigue; answering yes to the question: have you suffered any general fatigue/tiredness over the last three months? ${ }^{25}$ Weight loss; answering yes to the question: have you suffered from any weight loss over the last three months?15 Low physical activity was defined as 1-2 walks / week or less. Low balance; 47 or lower at Bergs balance scale. ${ }^{16}$ Low gait speed; walking four meters in $6.8 \mathrm{sec}$ onds or slower. 17 Visual impairment; a visual acuity of $\leq 0.5$ in both eyes, using KM chart. ${ }^{18}$

The study population was categorized from the number of frailty indicators (0-7) exceeding the cut off points. Persons not experiencing any frailty indicator were defined as not being frail; persons who exceeded the cutoff point on one or two frailty indicators were defined to be at risk of frailty, while persons whom exceeded the cut off points on three or more frailty indicators were defined as being frail.

\section{Statistical analysis}

The Chi-square test for independence was conducted to examine differences between groups based on gender, age, civil status and level of education in relation to life satisfaction. The Chi-square test for independence was also used to examine gender differences on every item in LiSat-11 and gender differences in life satisfaction in relation to sociodemographic data. Significant gender differences are presented in text, not in tables. When the variable used in a chi-square analysis could be ordered, linear by linear analysis was conducted. To control for confounders when looking for associations between sociodemographic data and life satisfaction, univariate analyses of covariance were conducted using the full scale of responses instead of categories. A multiple regression analysis was conducted to investigate to what extent the

variation in the global question on satisfaction with life as a whole could be explained by the ten underlying item scales in Fugl-Meyers assessment LiSat-11. The explained variance reported has been adjusted for the number of predictors and respondents. A linear regression analysis was performed to examine the association between frailty and life satisfaction. To further investigate how frailty affected the perceived life satisfaction a one-way ANOVA was conducted, and a Bonferoni analysis was made to control for the problem of multiple comparisons. Further, Dunett T3 post hoc

Table 1. Self-reported life satisfaction using Fugl-Meyer's LiSat-11, N= 577.

\begin{tabular}{|c|c|c|c|c|c|}
\hline & $\begin{array}{l}\text { Dissatisfied } \\
\text { (\%) }\end{array}$ & $\begin{array}{c}\text { Rather } \\
\text { satisfied }(\%)\end{array}$ & $\begin{array}{l}\text { Satisfied } \\
\text { (\%) }\end{array}$ & $\begin{array}{l}\text { Missing } \\
(\%)\end{array}$ & P* \\
\hline Life as a whole & 3 & 31 & 66 & & 0.000 \\
\hline Not frail & 0 & 16 & 84 & & \\
\hline At risk of frailty & 1 & 29 & 70 & & \\
\hline Frail & 9 & 42 & 48 & & \\
\hline Vocational situation & 8 & 31 & 59 & 2 & 0.000 \\
\hline Not frail & 0 & 22 & 78 & & \\
\hline At risk of frailty & 5 & 30 & 65 & & \\
\hline Frail & 19 & 38 & 43 & & \\
\hline Financial situation & 3 & 24 & 73 & 0 & 00.35 \\
\hline Not frail & 2 & 29 & 69 & & \\
\hline At risk of frailty & 3 & 22 & 75 & & \\
\hline Frail & 5 & 26 & 69 & & \\
\hline Leisure & 9 & 24 & 66 & 1 & 0.000 \\
\hline Not frail & 2 & 14 & 84 & & \\
\hline At risk of frailty & 6 & 23 & 71 & & \\
\hline Frail & 19 & 32 & 49 & & \\
\hline Contacts with friends & 7 & 18 & 76 & 0 & 0.002 \\
\hline Not frail & 2 & 21 & 77 & & \\
\hline At risk of frailty & 5 & 18 & 77 & & \\
\hline Frail & 14 & 15 & 71 & & \\
\hline Sexual life & 20 & 11 & 27 & $42 * *$ & 00.16 \\
\hline Not frail & 26 & 9 & 65 & & \\
\hline At risk of frailty & 33 & 22 & 45 & & \\
\hline Frail & 39 & 18 & 43 & & \\
\hline ADL & 3 & 13 & 84 & 0 & 0.000 \\
\hline Not frail & 0 & 3 & 97 & & \\
\hline At risk of frailty & 1 & 10 & 89 & & \\
\hline Frail & 7 & 25 & 68 & & \\
\hline Family life & 5 & 7 & 83 & 6 & 0.037 \\
\hline Not frail & 0 & 4 & 96 & & \\
\hline At risk of frailty & 4 & 7 & 89 & & \\
\hline Frail & 9 & 9 & 82 & & \\
\hline Partnership relation & 1 & 4 & 43 & $52^{* *}$ & 00.37 \\
\hline Not frail & 0 & 9 & 91 & & \\
\hline At risk of frailty & 4 & 8 & 88 & & \\
\hline Frail & 0 & 5 & 95 & & \\
\hline Physical health & 11 & 32 & 57 & 0 & 0.000 \\
\hline Not frail & 0 & 17 & 83 & & \\
\hline At risk of frailty & 7 & 29 & 63 & & \\
\hline Frail & 24 & 42 & 34 & & \\
\hline Psychological health & 3 & 19 & 77 & 0 & 0.000 \\
\hline Not frail & 0 & 9 & 91 & & \\
\hline At risk of frailty & 2 & 16 & 82 & & \\
\hline Frail & 6 & 31 & 63 & & \\
\hline
\end{tabular}

${ }^{*}$ Chi square test for independence was used to examine if frailty status affected perceived life satisfaction. ${ }^{* *}$ The high percentage of miss-

ing responses was due to the high proportion reporting not to have a partner. 
analysis was made to investigate where the differences were. IBM SPSS version 20 has been used; level of significance was set to $<0.05$ in all statistical tests conducted in this study.

\section{Results}

Within the study population ( $\mathrm{n}=577)$ the majority reported to be satisfied with life as a whole. The response rate on LiSat-11 was good with the exception of two items; sexual life and partner relationship, where barely half of the study population responded (Table 1). This could partly be explained by the large percentage missing a partner due to widowhood.

\section{Frailty and life satisfaction}

The non-frail respondents reported to be satisfied with life as a whole to a higher extent than the respondents categorized as frail (Table 1). Most life satisfaction items were significantly associated with frailty status, with non-frail participants being satisfied to a higher extent for all items with the exception of financial situation, sexual life and partnership relation. There was a significant difference in the odds for not being satisfied with life as a whole (Table 2). Compared to those not being frail, the frail respondents had an OR of 5.8 and those at risk of frailty had an OR of 2.3. No significant gender differences in reported life satisfaction were found between the three categories based on grade of frailty. Within this study population neither age nor level of education significantly affected the level of frailty. Linear regression analysis showed that $7.2 \%$ of the variance in life satisfaction can be explained by frailty and that an increased frailty indicator by one means a decrease in life satisfaction with 0.27 units (Pearson correlation, $\mathrm{P}<0.001)$.

A one-way ANOVA analysis confirmed a decrease in life satisfaction between the three categories of frailty used in this study (Figure 1). Based on Bonferroni post hoc analysis, three item scales out of the eleven that constitute Fugl-Meyer's assessment, were excluded due to high risk of type 1 error, these were financial situation, sexual life and partnership relation. Respondents categorized as at risk of frailty reported lower life satisfaction than the non-frail respondents, and respondents categorized as frail reported lower life satisfaction than the respondents at risk of frailty.

The difference in satisfaction between the non-frail and the frail was significant on all items included in the ANOVA analysis. Between the non-frail and those at risk of frailty the difference in satisfaction was significant except for the items on life as a whole and contacts with friends. The difference in satisfaction between those at risk of frailty and the already frail was significant except for the item on family life.

\section{Socio-demographic data}

Men were to a significantly higher extent satisfied with life as a whole and with their physical health than were women, while men were significantly less satisfied with their sexual life than were women. Over $70 \%$ of the men were married or cohabiting compared with barely $30 \%$ among women. To be married and to be living together with someone were positively associated with a higher life satisfaction within the study population. The gender separated analysis showed that the civil status significantly correlated with life satisfaction among women. Married women reported to have higher life satisfaction than did women with another civil status.
Age and level of education did not significantly affect the perceived satisfaction with life as a whole. The distribution in life satisfaction within the socio-demographic categories; gender, age, civil status, living situation and education, can be seen in Table 3. We also analyzed life satisfaction in the socio-demographic subgroups by frailty status, with significant associations between frailty status and life satisfaction in all subgroups with the exception of age $>90$ and unmarried/living apart (data not shown).

\section{LiSat-11}

Multiple regression analysis showed that the ten underlying items in LiSat-11 explained $42.6 \%$ of the variance in satisfaction with life as a whole. Out of these ten underlying items, four items significantly explained life satisfaction. These were in order (the factor with

Table 2. Odds ratio (OR) for not being satisfied $\left(^{*}\right)$ in the three frailty categories, with 95\% confidence interval (CI) and P-value Not frail as reference group. $\mathrm{N}=577$.

\begin{tabular}{lccc} 
& OR & CI & P \\
Not frail & 1 & & \\
At risk of frailty & 2.3 & $1.10-4.89$ & 0.027 \\
\hline Frail & 5.8 & $2.67-12.58$ & 0.000 \\
\hline
\end{tabular}

*Dissatisfied (1-3) or rather satisfied (4) at the scale of percieved satisfaction with life as a whole (1-6), LiSat.

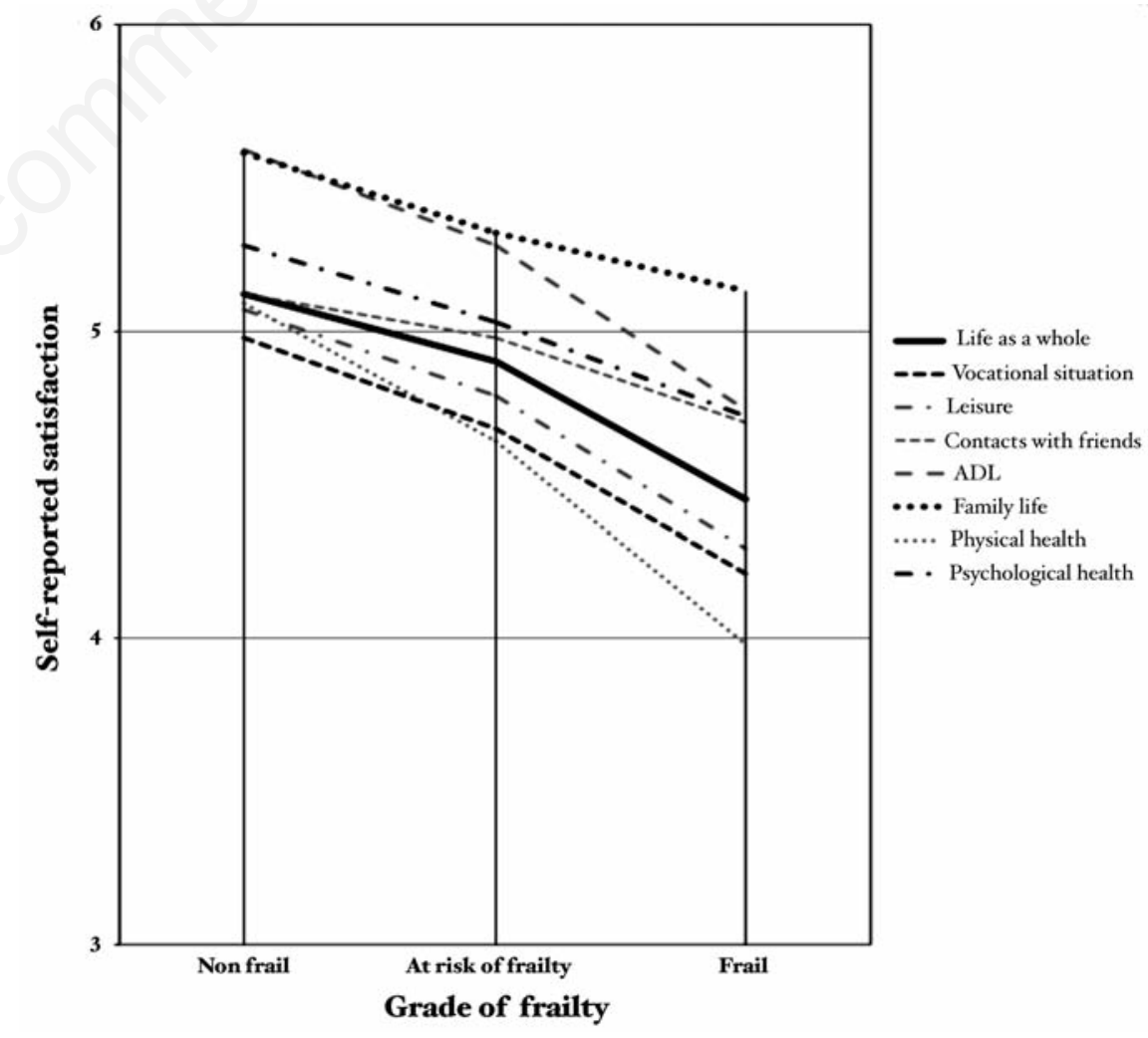

Figure 1. Self-reported satisfaction: 1 = very dissatisfied, 2 = dissatisfied, 3 = rather dissatisfied, 4 = rather satisfied, 5 = satisfied, 6 = very satisfied, in relation to grade of frailty. 
highest value first): psychological health, partner relationship, leisure and ADL. All four items were positively correlated with life satisfaction, for instance with an increased unit in weighted satisfaction on psychological health, life satisfaction increased with 0.18 units $(\max 6)$.

\section{Discussion}

This study shows that satisfaction with life as a whole among older adults is almost as high as in younger age groups. In fact, for those not being frail it was even higher than for younger adults, while those being frail had lower life satisfaction. Satisfaction with life as a whole was, in this older population, significantly associated with satisfaction with psychological health, partnership relationship, leisure and ADL.

A large proportion of the study population considered themselves as satisfied with life and with the most of the underlying items in LiSat-11. 66\% reported to be satisfied with life as a whole which is almost the same level as has been reported in a working age population.9,19,20 Family life and ADL were the items with highest rate of satisfaction. The psychological health was generally satisfactory and distinguished from the satisfaction with the physical health that was rated markedly lower. There was a high proportion of missing responses to the question about satisfaction with sexual life. Still, $48 \%$ have answered and almost $30 \%$ considered themselves as satisfied with their sexual life. According to a study addressing 70 years olds in Gothenburg, two thirds of men and women reported to be satisfied with their sexual life. ${ }^{21}$ The lower grade of satisfaction in the present study may depend on the higher mean age and the high proportion of respondents (mostly women) not having a partner in life.

Those not being frail had even higher levels of satisfaction with life as a whole ( $85 \%$ being satisfied) compared to younger age groups (70\%), ${ }^{19}$ those at risk of frailty had about the same satisfaction as younger age groups (70\%), while those being frail had lower satisfaction (48\%). This was also true for the other dimensions of frailty with the exception of financial situation, sexual life and partnership relation. Thus, there was an association between frailty and life satisfaction in this sample. This can be interpreted as it is not the increasing age that cause the deterioration in life satisfaction as much as the increasing frailty. Stereotypes and attitudes toward older adults are mostly negative, but it is known that detailed information can affect the attitudes to be less negative. ${ }^{22}$ Information about frailty status ought to nuance the stereotypes of aging, which our results indicate.

In accordance with previous research addressing older adults, ${ }^{6}$ gender differences were found, men reported higher life satisfaction than women. In a working age population only marginal gender differences in life satisfaction were found, ${ }^{19}$ while life satisfaction has been proven to be gender independent in other studies.9,20 Gender differences may vary with generation affiliation and situational fac- tors connected to age. To be married was positively correlated with life satisfaction among women within the sample. Women reported widowhood and to live alone to a higher extent than men, which may partly explain the lower self-perceived life satisfaction among women within this study. In general women still have less material resources than men, which also can affect life satisfaction through lower standard of living and through economic worries. ${ }^{23}$

There were no significant age differences in life satisfaction or in grade of frailty among the respondents. To examine the effect of age on life satisfaction and frailty a wider age range is desired. The interaction between gender and education was probably too similar to distinguish the effect of education itself on life satisfaction within this sample.

As in previous studies addressing older adults, ${ }^{10,24,25}$ satisfaction with the ADL-situation was a significant factor explaining the variance in life satisfaction. Even though only four items in LiSat-11 proved to be significant as explanatory factors to life satisfaction, the other items still represent important components in the respondents' lives; components that were reported to worsen with an increased degree of frailty.

Satisfaction with both ADL and physical health worsened with increased frailty, but the physical health was reported to be worse than the ADL situation. This may be a result of the inclusion criteria to the larger of the two samples on which this study is based; to be independent of home help service or help with activities of daily living. In rehabilitation research health has been defined as being able

Table 3. The study population divided into categories of self-perceived life satisfaction in relation to socio-demographic data, presented in \%.

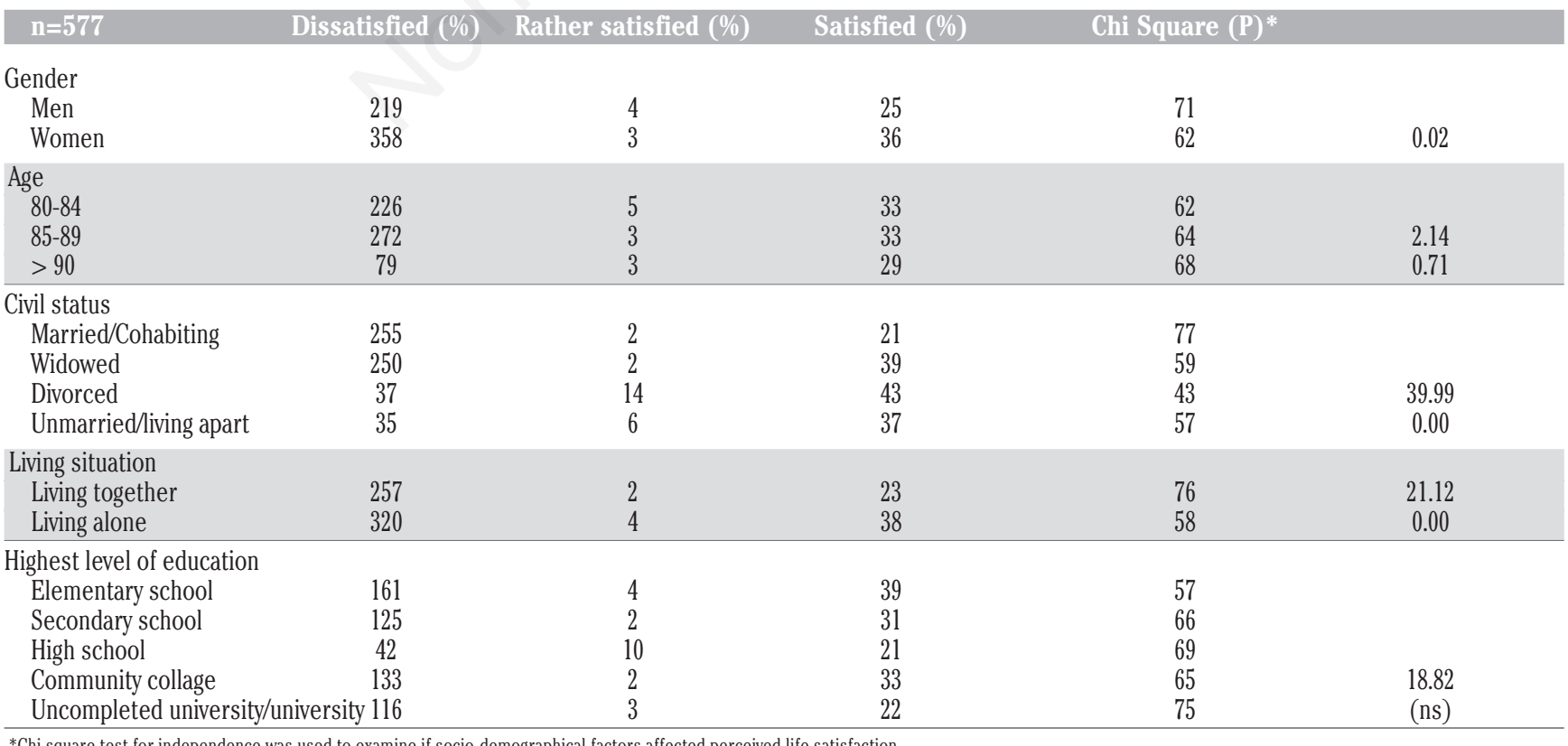

*Chi square test for independence was used to examine if socio-demographical factors affected perceived life satisfaction. 
to fulfill individual, realistic goals, ${ }^{9}$ which is why life satisfaction has been more commonly expressed among older adults occupied as usual and independent of home service. ${ }^{10}$ The rather high level of satisfaction with ADL could indicate that this sample still may be able to fulfill their goals, despite a worsened physical health. That may also partly explain the rather high level of satisfaction with life as a whole.

$\mathrm{ADL}$ and leisure are factors of importance to self-perceived life satisfaction and are factors affected by frailty. Life satisfaction was reported to be significantly higher among the nonfrail respondents, than among respondents at risk of frailty and the frail. This transition could be reconciled with an experience of crisis, and a decrease in life satisfaction, while entering the next and last phase in life. ${ }^{26}$ The linear regression analysis showed that frailty explained $7 \%$ of the variance in satisfaction with life as a whole. This stresses the need for interventions with the purpose of preventing or delaying frailty among older adults in order to maintain high levels of life satisfaction.

Studies have shown that older adults who are at risk of frailty or who are frail, but are not yet disabled, benefit the most from health promoting interventions. ${ }^{2}$ The gender differences found in physical health and in the overall perceived life satisfaction indicate a complexity and a need of a gender perspective in interventions addressing older adults. More research is needed to support development of this kind of intervention.

The ten underlying items in LiSat-11 together explained $43 \%$ of the variance in satisfaction with life as a whole compared with $70 \%$ in a working age population. ${ }^{19}$ The difference in explained variance could indicate that the instrument was more sensitive within a younger population than within an older population. According to Neugarten, ${ }^{7}$ it is not appropriate to measure life satisfaction among older adults with the same instrument that is used within a population of working age while these instruments have references like vocational situation and social involvement, factors that may have declined with increasing age. On the question about vocational situation in this study the older adults were asked to refer to their daily chores, unlike leisure that refers to the activities of choice. The questions may have been difficult to distinguish but the response rate was high in both of them. The fact that the LiSat-11 has only been validated for ages up to 74 years is a limitation of this study. But neither for the younger age groups nor for our age group there was no association between satisfaction with life as a whole and age. 19

Non-participant rate tends to rise with increasing age, ${ }^{27}$ but was not considered to result in a selective sample in this study. The main reason not to participate in the present studies was due to no interest. A minor group reported not to have the strength to participate. ${ }^{11,12}$ The sample was considered representative since the target group was frail older adults, not too healthy and not too weak.

The LiSat-11 is a scale with categorical responses, with no information if the intervals between the different categories are equal, leading to difficulties interpreting results from continuous statistical tests. This is a limitation of the study, as well as breaking the scale down to three categories which might hide other effects than between these categories. On the other hand, it is with these categories the LiSat-11 is validated. ${ }^{9}$

\section{Conclusions}

Older adult people's satisfaction with life as a whole is almost as high as in younger age groups. It is significantly associated with satisfaction with psychological health, partnership relationship, leisure and ADL. Respondents with a higher degree of frailty reported significantly lower grades of life satisfaction, indicating a possibility of maintaining their life satisfaction by preventing or delaying the development of frailty.

\section{References}

1. Fried LP, Ferrucci L, Darer J, et al. Untangling the concepts of disability, frailty, and comorbidity: implications for improved targeting and care. J Gerontol A Biol Sci Med Sci 2004;59:255-63.

2. Ferrucci L, Guralnik JM, Studenski S, et al. Designing randomized, controlled trials aimed at preventing or delaying functional decline and disability in frail, older persons: a consensus report. J Am Geriatr Soc 2004;52:625-34.

3. Langlois F, Vu TTM, Kergoat MJ, et al. The multiple dimensions of frailty: physical capacity, cognition, and quality of life. Intl Psychogeriatr 2012;24:1429-36.

4. Richardson S, Karunananthan S, Bergman H. I may be frail but I ain't no failure. Can Geriatr J 2011;14:24-8.

5. Bowling A, Grundy E. Activities of daily living: changes in functional ability in three samples of elderly and very elderly people. Age Ageing 1997;26:107-14.

6. Borg C, Hallberg IR, Blomqvist K. Life satisfaction among older people (65+) with reduced self-care capacity: the relationship to social, health and financial aspects. J Clin Nurs 2006;15:607-18.

7. Neugarten BL, Havighurst RJ, Tobin SS. The measurement of life satisfaction. J
Gerontol 1961;16:134-43.

8. Anke AGW, Fugl-Meyer AR. Life satisfaction several years after severe multiple trauma - A retrospective investigation. Clin Rehabil 2003;17:431-42.

9. Fugl-Meyer AR, Bränholm I-B, Fugl-Meyer KS. Happiness and domain-specific life satisfaction in adult northern Swedes. Clin Rehabil 1991;5:25-33.

10. Johannesen A, Petersen J, Avlund K. Satisfaction in everyday life for frail 85 year-old adults: A Danish population study. Scand J Occup Ther 2004;11:3-11.

11. Dahlin-Ivanoff S, Gosman-Hedstrom G, Edberg AK, et al. Elderly persons in the risk zone. Design of a multidimensional, health-promoting, randomised threearmed controlled trial for prefrail people of $80+$ years living at home. BMC Geriatr 2010;10:27.

12. Wilhelmson K, Duner A, Eklund K, et al. Design of a randomized controlled study of a multi-professional and multidimensional intervention targeting frail elderly people. BMC Geriatr 2011;11:24.

13. Folstein MF FS, McHugh PR. Mini-mental state. A practical method for grading the cognitive state of patients for the clinician. J Psychiatr Res 1975;12:189-98.

14. Mathiowetz V, Kashman N, Volland G, et al. Grip and pinch strength: normative data for adults. Arch Phys Med Rehabil 1985;66:69-74.

15. Tibblin G, Tibblin B, Peciva S, et al. The Goteborg quality of life instrument: an assessment of well-being and symptoms among men born 1913 and 1923. Methods and validity. Scand J Prim Health Care Suppl 1990;1:33-8.

16. Chiu AY, Au-Yeung SS, Lo SK. A comparison of four functional tests in discriminating fallers from non-fallers in older people. Disabil Rehabil 2003;25:45-50.

17. Peterson MJ, Giuliani C, Morey MC, et al. Physical activity as a preventative factor for frailty: the health, aging, and body composition study. J Gerontol A Biol Sci Med Sci 2009;64:61-8.

18. Moutakis K, Stigmar G, Hall-Lindberg J. Using the KM visual acuity chart for more reliable evaluation of amblyopia compared to the HVOT method. Acta Ophthalmol Scan 2004;82:547-51.

19. Fugl-Meyer AR, Melin R, Fugl-Meyer KS. Life satisfaction in 18- to 64-year-old Swedes: In relation to gender, age, partner and immigrant status. J Rehabil Med 2002;34:239-46.

20. Melin R, Fugl-Meyer KS, Fugl-Meyer AR Life satisfaction in 18- to 64-year-old Swedes: in relation to education, employment situation, health and physical activity. Journal Rehabil Med 2003;35:84-90.

21. Beckman N, Waern M, Gustafson D, Skoog 
I. Secular trends in self reported sexual activity and satisfaction in Swedish 70 year olds: cross sectional survey of four populations, 1971-2001. BMJ 2008;337: a279.

22. Kite ME, Stockdale GD, Whitley Jr BE, Johnson BT. Attitudes toward younger and older adults: An updated meta-analytic review. J Soc Issues 2005;61:241-66.

23. Pinquart M, Sorensen S. Gender differ- ences in self-concept and psychological well-being in old age: a meta-analysis. J Gerontol B Psychol Sci Soc Sci 2001;56:P195-213.

24. Berg AI, Hassing LB, McClearn GE, Johansson B. What matters for life satisfaction in the oldest-old? Aging Ment Health 2006;10:257-64.

25. Hilleras PK, Jorm AF, Herlitz A, Winblad B. Life satisfaction among the very old: A sur- vey on a cognitively intact sample aged 90 years or above. Int $\mathrm{J}$ Aging Hum Dev 2001;52:71-90.

26. Fillit H, Butler RN. The frailty identity crisis. J Am Geriatr Soc 2009;57:348-52.

27. Ridda I, Lindley R, MacIntyre RC. The challenges of clinical trials in the exclusion zone: the case of the frail elderly. Australas J Ageing 2008;27:61-6. 